\title{
Isospin relaxation time in heavy-ion collisions at intermediate energies
}

\author{
Bao-An Li and C. M. Ko \\ Cyclotron Institute and Department of Physics, Texas A\&M University, College Station, Texas 77843
}

(Received 12 November 1997)

\begin{abstract}
Using an isospin-dependent transport model, we have studied the isospin and momentum relaxation times in the heavy residues formed in heavy-ion collisions at intermediate energies. It is found that only at incident energies below the Fermi energy can chemical or thermal equilibrium be reached before dynamical instability is developed in the heavy residues. Also, the isospin relaxation time is shorter (longer) than that for momentum at beam energies lower (higher) than the Fermi energy. [S0556-2813(98)05204-2]
\end{abstract}

PACS number(s): 25.70.Lm, 25.70.Mn, 24.10.Nz

In studying heavy-ion collisions at intermediate energies, it is important to know if thermodynamical, i.e., thermal (momentum) and chemical (isospin), equilibrium can be reached in the heavy residues formed in these collisions. Although the early stage of heavy-ion collisions must be described by microscopic models, a macroscopic treatment of the later stage in terms of temperature, volume, and chemical potential becomes possible if thermodynamical equilibrium is established. Also, the interpretation of nuclear multifragmentation in heavy-ion collisions either as a dynamical or as a statistical process depends on whether thermodynamical equilibrium is achieved in the collisions. Based on the assumption that thermodynamical equilibrium can be established in heavy-ion collisions, many statistical models have been developed, and they seem to be quite successful in describing the experimental data. A critical examination of thermodynamical equilibrium in heavy-ion collisions is thus needed. A review on this subject can be found in Ref. [1].

In the past few years, extensive theoretical efforts have been devoted to investigate the rate of thermalization of nuclear matter in intermediate-energy heavy-ion collisions. Using the Uehling-Uhlenbeck equation, momentum relaxation both in collisions of semi-infinite nuclear matter and in infinite nuclear matter have been studied [2-4]. Collisions of finite nuclei have also been studied using the BoltzmannUehling-Uhlenbeck equation [5-8]. On the other hand, only limited studies have been carried out for understanding the rate of chemical equilibrium in heavy-ion collisions $[9,10]$. In both statistical and dynamical models it is usually assumed that chemical equilibrium is reached either instantaneously or as fast as thermal equilibrium. This is supported by deep-inelastic heavy-ion collisions at low energies, where the isospin degree of freedom has been found to first reach equilibrium [11-13]. Recent experimental studies [14-17] of the isotopic composition of intermediate-mass fragments (IMF's) and their angular distributions in heavy-ion collisions have also shown that only at low energies $(\approx 30 \mathrm{MeV} /$ nucleon) is isospin equilibrium reached before their emission.

With recent advances in experiments using radioactive beams with large neutron or proton excess, it has been possible to create a transient state of nuclear matter with appreciable isospin asymmetry. Knowledge of the rate of chemical and thermal equilibrium in such an asymmetric nuclear matter is thus needed [18].
In this Brief Report, we shall report the results from a study of comparing the rate of chemical equilibrium to that of thermal equilibrium using an isospin-dependent Boltzmann-Uehling-Uhlenbeck (BUU) transport model. The model has been quite successful in predicting and explaining a number of isospin-dependent phenomena in heavy-ion collisions at intermediate energies [9,19-22]. The isospin dependence is introduced in the model through using different total and differential cross sections, Pauli blocking, and mean fields for protons and neutrons. A recent review of this model, especially its isospin-dependent aspects, is given in Ref. [18].

In the model study of heavy-ion collisions, a heavy residue can be identified as a collection of nucleons with densities higher than 1/10 of normal nuclear matter density. To characterize the degree of chemical equilibrium, we introduce the quantity

$$
\lambda_{I}(t) \equiv \frac{(n / p)_{y>0}}{(n / p)_{y<0}},
$$

where $(n / p)_{y>0}$ and $(n / p)_{y<0}$ are, respectively, the neutron to proton ratio for positive and negative rapidity nucleons in the rest frame of the residue. If chemical equilibrium is established, this quantity then has a value of 1 . We further define the isospin relaxation time $\tau_{I}$ as the time when the quantity $\left[\lambda_{I}(t)-1\right] /\left[\lambda_{I}(0)-1\right]$ is 0.01 ; i.e., it is $1 \%$ from its equilibrium value. This time is an approximate measure of the rate at which the residue reaches chemical equilibrium. We note that this definition is somewhat different from that one would usually use, i.e., $\lambda\left(\tau_{I}\right)=1 / e$.

For describing thermal equilibrium of the heavy residue, we use the quadrupole moment $Q_{z z}(t)$ in its rest frame, i.e.,

$$
Q_{z z}(t)=\int \frac{d \vec{r} d \vec{p}}{(2 \pi)^{3}}\left(2 p_{z}^{2}-p_{x}^{2}-p_{y}^{2}\right) f(\vec{r}, \vec{p}, t),
$$

where $f(\vec{r}, \vec{p}, t)$ is the Wigner function from the BUU model calculations. Obviously $Q_{z z}=0$ is a necessary, although not a sufficient, condition for thermal equilibrium. Similar to the definition of $\tau_{I}$ we define the momentum relaxation time $\tau_{p}$ as the time when $Q_{z z}(t) / Q_{z z}(0)=1 \%$. This quantity then measures the rate at which the residue reaches thermal equilibrium. 


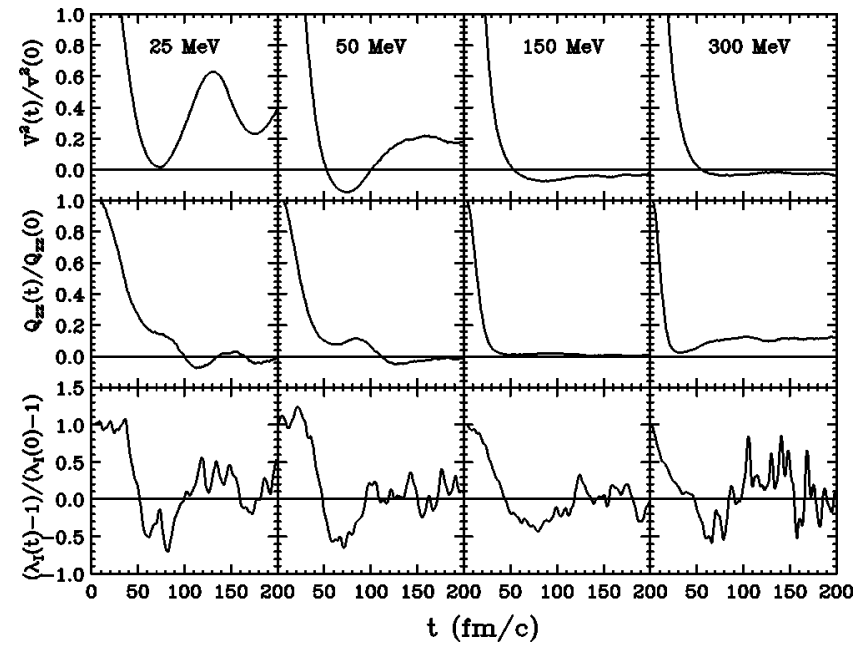

FIG. 1. The development of dynamical instability (upper windows), thermal (middle windows), and chemical (lower windows) equilibrium in ${ }^{40} \mathrm{Ca}+{ }^{124} \mathrm{Sn}$ collisions at an impact parameter of 1 $\mathrm{fm}$ and beam energies of $25,50,150$, and $300 \mathrm{MeV} /$ nucleon, respectively.

Another important property of the heavy residue is possible existence of dynamical instability. To study this phenomenon, we introduce the square of the adiabatic sound velocity $[23,24]$,

$$
v_{s}^{2}=\frac{1}{m}\left(\frac{\partial P}{\partial \rho}\right)_{S}=\frac{1}{m}\left[\frac{10}{9}\left\langle E_{k}\right\rangle+a \frac{\rho}{\rho_{0}}+b \sigma\left(\frac{\rho}{\rho_{0}}\right)^{\sigma}\right],
$$

where $\left\langle E_{k}\right\rangle$ is the average kinetic energy per nucleon, $a=-358.1 \mathrm{MeV}, b=304.8 \mathrm{MeV}$, and $\sigma=7 / 6$ are the parameters corresponding to a soft nuclear equation of state. For $v_{s}^{2}<0$, a homogeneous nuclear matter is unstable against the growth of fluctuation, leading to dynamical instability or spinodal decomposition.

We have studied the time dependence of $\left[\lambda_{I}(t)\right.$ $-1] /\left[\lambda_{I}(0)-1\right]$ and $Q_{z z}(t) / Q_{z z}(0)$ compared to that of $v_{s}^{2}(t) / v_{s}^{2}(0)$. They are shown in Fig. 1 for collisions of ${ }^{40} \mathrm{Ca}+{ }^{124} \mathrm{Sn}$ at an impact parameter of $1 \mathrm{fm}$ and at beam energies of 25, 50, 150, and $300 \mathrm{MeV} /$ nucleon. The system considered here has an initial $\lambda_{I}(0)=1.48$. For collisions at a beam energy of $25 \mathrm{MeV} /$ nucleon the residue is found to be dynamically stable up to $300 \mathrm{fm} / c$ during the collision. This time interval is long enough for thermal and chemical equilibrium to be fully established as shown in the middle and lower windows of the first column. On the other hand, for collisions at beam energies above $50 \mathrm{MeV} /$ nucleon a significant compression appears, and this is followed by an expansion, leading into the adiabatic spinodal region after about 50 $\mathrm{fm} / c$. We notice that at this time the heavy residue formed in the collision is still far from thermal and chemical equilibrium. Both the momentum and isospin asymmetries of the heavy residue are seen to change oscillatorily with time. The study of the nature of this oscillation in isospin asymmetry, in particular its possible connection to the giant dipole resonance in nuclei, is underway and will be reported elsewhere.

We also notice from the middle window of the fourth column that nuclear transparency occurs at $E / A=300 \mathrm{MeV}$. After spinodal decomposition the heavy residue quickly

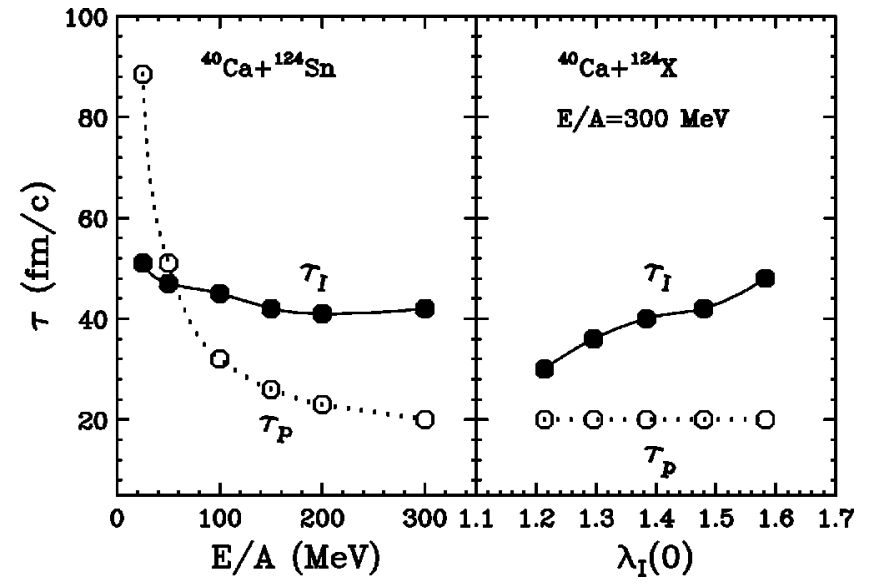

FIG. 2. Left panel: isospin (open circles) and momentum (solid circles) relaxation times as functions of beam energy for the reactions shown in Fig. 1. Right panel: relaxation times as functions of $\lambda_{I}(0)$ of the reactions.

starts to break up into fragments and nucleons [24]. Although the isotopic content of these fragments and nucleons depends on the emission angle, it is also strongly influenced by the neutron to proton ratio of the target and projectile in the entrance channel instead of the average neutron to proton ratio of the combined system. This observation is consistent with recent experimental findings [14-17].

Although chemical and thermal equilibrium is not completely established at beam energies higher than the Fermi energy, it is still interesting to compare its relaxation times. This is shown in Fig. 2. In the left window, the comparison is made for ${ }^{40} \mathrm{Ca}+{ }^{124} \mathrm{Sn}$ collisions at beam energies from 25 to $300 \mathrm{MeV} /$ nucleon and at an impact parameter of $1 \mathrm{fm}$. The momentum relaxation time is found to decrease with increasing beam energy. This is in qualitative agreement with that found in Refs. [2-6] and [8]. On the other hand, the isospin relaxation time decreases slowly with the beam energy. The shorter isospin relaxation time at incident energies below about $50 \mathrm{MeV} /$ nucleon is in agreement with what was found in deep-inelastic heavy-ion collisions [11-13]. At higher incident energies the time for reaching momentum equilibrium is found to be shorter than that for isospin equilibrium. For example, a $20 \mathrm{fm} / c$ difference in the relaxation time is observed at $E / A=300 \mathrm{MeV}$. In the right window, the isospin and momentum relaxation times as functions of the initial isospin asymmetry $\lambda_{I}(0) \equiv(n / p)_{\text {projectile }} /(n / p)_{\text {target }}$ are compared for ${ }^{40} \mathrm{Ca}$-induced reactions on several isobaric targets of mass 124 at a beam energy of $300 \mathrm{MeV}$ and at an impact parameter of $1 \mathrm{fm}$. It is seen that although the momentum relaxation is almost independent of the initial isospin asymmetry, the isospin relaxation time increases with the initial isospin asymmetry.

The short relaxation time for isospin than momentum at low incident energies can be understood as follows. First, nucleon-nucleon collisions, which are responsible for momentum relaxation, are more likely to be suppressed due to Pauli blocking. Second, the repulsive symmetry potential for neutrons and the attractive symmetry potential for protons make preequilibrium emissions of neutrons more likely than protons in low-energy collisions, as shown in Ref. [22], which thus enhances the isospin relaxation rate in the resi- 
due. On the other hand, in high-energy collisions, Pauli blocking is less effective and the symmetry potential is also less important, leading thus to a shorter momentum relaxation time and a longer isospin relaxation time.

We have also studied effects due to different forms of the symmetry potential and the charge exchange reaction ( $p n$ $\rightarrow n p$ ) on chemical and thermal equilibrium. They are found to have no discernible effects on both the momentum and isospin relaxation times. Only during the later stage of the collisions do they affect slightly the momentum and isospin distributions.

In summary, using an isospin-dependent BUU model we have studied the isospin and momentum relaxation times in the heavy residues formed in heavy-ion collisions at interme- diate energies. They are compared both with each other and also with the time for dynamical instability to appear in the heavy residue. We have found that chemical and thermal equilibrium can be completely established only at beam energies below the Fermi energy. At higher energies the dynamical instability sets in before either chemical or thermal equilibrium is achieved. Moreover, the isospin relaxation time is shorter (longer) than that for momentum at beam energies lower (higher) than the Fermi energy.

This work was supported in part by the NSF Grant No. PHY-9509266 and the Robert A. Welch Foundation under Grant No. A-1358.
[1] L. G. Moretto and G. J. Wozniak, Annu. Rev. Nucl. Part. Sci. 43, 123 (1993).

[2] G. F. Bertsch, Z. Phys. A 289, 103 (1978).

[3] J. Randrup, Nucl. Phys. A314, 429 (1979).

[4] C. Toepffer and C. Y. Wong, Phys. Rev. C 25, 1018 (1982).

[5] W. Cassing, Z. Phys. A 465, 317 (1987).

[6] P. Abgrall et al., Phys. Rev. C 49, 1040 (1994).

[7] F. Haddad et al., Z. Phys. A 354, 321 (1996).

[8] B. Borderie et al., Z. Phys. A 357, 7 (1997).

[9] B. A. Li and S. J. Yennello, Phys. Rev. C 52, R1746 (1995).

[10] R. Donangelo and S. R. Souza, Phys. Lett. B 409, 58 (1997).

[11] B. Gatty et al., Z. Phys. A 273, 65 (1975).

[12] F. Beck, M. Dworzecka, and H. Feldmeier, Z. Phys. A 289, 113 (1978).

[13] W. U. Schröder and J. R. Huizenga, in Treatise on Heavy-Ion Science, edited by D. A. Bromley (Plenum, New York, 1984), Vol. 2, p. 113.
[14] S. J. Yennello et al., Phys. Lett. B 321, 15 (1994).

[15] H. Johnston et al., Phys. Lett. B 371, 186 (1996).

[16] H. Johnston et al., Phys. Rev. C 56, 1972 (1997).

[17] E. Ramakrishnan et al., Phys. Rev. C (to be published).

[18] B. A. Li, C. M. Ko, and W. Bauer, Int. J. Mod. Phys. E (to be published).

[19] B. A. Li, Z. Z. Ren, C. M. Ko, and S. J. Yennello, Phys. Rev. Lett. 76, 4492 (1996).

[20] R. Pak et al., Phys. Rev. Lett. 78, 1022 (1997).

[21] R. Pak et al., Phys. Rev. Lett. 78, 1026 (1997).

[22] B. A. Li, C. M. Ko, and Z. Z. Ren, Phys. Rev. Lett. 78, 1644 (1997).

[23] W. Bauer, G. F. Bertsch, and H. Schultz, Phys. Rev. Lett. 69, 1888 (1992).

[24] B. A. Li and D. H. E. Gross, Nucl. Phys. A554, 257 (1993). 